\title{
A new blow up criterion for the 3D magneto-micropolar fluid flows without magnetic diffusion
}

\section{Dongxiang Chen ${ }^{1 *}$ and Qifeng Liu'}

\section{"Correspondence:}

chendx020@163.com

${ }^{1}$ School of Mathematics and

Statistics, Jiangxi Normal University,

Nanchang, China

\section{Springer}

\begin{abstract}
This note obtains a new regularity criterion for the three-dimensional magneto-micropolar fluid flows in terms of one velocity component and the gradient field of the magnetic field. The authors prove that the weak solution $(u, \omega, b)$ to the magneto-micropolar fluid flows can be extended beyond time $t=T$, provided if $u_{3} \in L^{\beta}\left(0, T ; L^{\alpha}\left(R^{3}\right)\right)$ with $\frac{2}{\beta}+\frac{3}{\alpha} \leq \frac{3}{4}+\frac{1}{2 \alpha}, \alpha>\frac{10}{3}$ and $\nabla b \in L^{\frac{4 p}{3(p-2)}}\left(0, T ; \dot{M}_{p, q}\left(R^{3}\right)\right)$ with $1<q \leq p<\infty$ and $p \geq 3$.

MSC: Primary 35Q35; secondary 76D03

Keywords: Magneto-micropolar fluid flow; Regularity criterion; Weak solution; Morrey spaces
\end{abstract}

\section{Introduction}

The aim of this paper is to understand the regularity criterion for the following threedimensional magneto-micropolar fluid flows without magnetic diffusion:

$$
\begin{cases}\partial_{t} u-(\mu+\chi) \Delta u+\nabla \pi=-u \cdot \nabla u+b \cdot \nabla b-\chi \nabla \times \omega, & (x, t) \in R^{3} \times(0, T), \\ \partial_{t} \omega-\gamma \Delta \omega-\kappa \nabla \operatorname{div} \omega+2 \chi \omega=-u \cdot \nabla \omega+\chi \nabla \times u, & (x, t) \in R^{3} \times(0, T), \\ \partial_{t} b+u \cdot \nabla b=b \cdot \nabla u, & (x, t) \in R^{3} \times(0, T), \\ \nabla \cdot u=\nabla \cdot b=0, & (x, t) \in R^{3} \times(0, T), \\ \left.u\right|_{t=0}=u_{0},\left.\quad \omega\right|_{t=0}=\omega_{0},\left.\quad b\right|_{t=0}=b_{0}, & x \in R^{3} .\end{cases}
$$

This system is a special case of the classical three-dimensional magneto-micropolar fluid flows

$$
\begin{cases}\partial_{t} u-(\mu+\chi) \Delta u+\nabla \pi=-u \cdot \nabla u+b \cdot \nabla b-\chi \nabla \times \omega, & (x, t) \in R^{3} \times(0, T), \\ \partial_{t} \omega-\gamma \Delta \omega-\kappa \nabla \operatorname{div} \omega+2 \chi \omega=-u \cdot \nabla \omega+\chi \nabla \times u, & (x, t) \in R^{3} \times(0, T), \\ \partial_{t} b+u \cdot \nabla b-v \Delta b=b \cdot \nabla u, & (x, t) \in R^{3} \times(0, T), \\ \operatorname{div} u=\operatorname{div} b=0, & (x, t) \in R^{3} \times(0, T), \\ \left.u\right|_{t=0}=u_{0},\left.\quad \omega\right|_{t=0}=\omega_{0},\left.\quad b\right|_{t=0}=b_{0}, & x \in R^{3},\end{cases}
$$

(c) The Author(s) 2021. This article is licensed under a Creative Commons Attribution 4.0 International License, which permits use sharing, adaptation, distribution and reproduction in any medium or format, as long as you give appropriate credit to the original author(s) and the source, provide a link to the Creative Commons licence, and indicate if changes were made. The images or other third party material in this article are included in the article's Creative Commons licence, unless indicated otherwise in a credit line to the material. If material is not included in the article's Creative Commons licence and your intended use is not permitted by statutory regulation or exceeds the permitted use, you will need to obtain permission directly from the copyright holder. To view a copy of this licence, visit http://creativecommons.org/licenses/by/4.0/. 
where $u=\left(u_{1}, u_{2}, u_{3}\right), \omega=\left(\omega_{1}, \omega_{2}, \omega_{3}\right), b=\left(b_{1}, b_{2}, b_{3}\right)$ and $\pi$ denote the unknown velocity field, the micro-rotational velocity, the magnetic field and the unknown scalar pressure at the point $(x, t) \in \mathbf{R}^{3} \times(0, T)$, respectively. While $u_{0}, \omega_{0}, b_{0}$ are the prescribed initial data and $\operatorname{div} u=\operatorname{div} b=0$ in the sense of distributions. The constants $\mu, \chi, \kappa, \gamma$ are positive numbers associated with the properties of the material, where $\mu$ is the kinematic viscosity, $\chi$ is the vortex viscosity, $\kappa$ and $\gamma$ are spin viscosities (more details see [11]).

The magneto-micropolar fluid system (1.2) was first proposed by Galdi and Rionero [7]. The existence of global-in-time weak solutions was then established by Rojas-Medar and Boldrini [14], while the local strong solutions and global strong solutions in bounded domain for the small initial data were considered, respectively, by Rojas-Medar [13] and Ortega-Torres and Rojas-Medar [12]. However, whether the local strong solutions can exist globally or the global weak solution is regular and unique is an outstanding open problem. Hence there are many regularity criteria to ensure the smoothness of solutions. Gala [2] proved that, if $u \in L^{\frac{2}{1-r}}\left(0, T ; \dot{M}_{p, \frac{3}{r}}\left(R^{3}\right)\right)$ or $\nabla u \in L^{\frac{2}{2-r}}\left(0, T ; \dot{M}_{p, \frac{3}{r}}\left(R^{3}\right)\right)$, then the local smooth solution $(u, \omega, b)$ can be extended beyond $t=T$. Zhang and Yao [17] demonstrated that, if $\nabla u \in L^{p}\left(0, T ; \dot{F}_{q, \frac{2 q}{3}}^{0}\left(R^{3}\right)\right)$ with $\frac{2}{p}+\frac{3}{q}=2, \frac{3}{2}<q \leq \infty$, then the weak solution $(u, \omega, b)$ is smooth on $[0, T]$.

When the micro-rotational velocity $\omega=0$ and $\chi=0$, Eq. (1.2) becomes the standard magneto-hydrodynamic equations. In recent years, the problem of regularity criteria involving one component has been investigated for the MHD equations (see e.g. [3-5, 8, 9]). In 2016, Yamazaki [15] proved that, if

$$
u_{3} \in L^{p}\left(0, T ; L^{q}\left(R^{3}\right)\right)
$$

with $\frac{2}{p}+\frac{3}{q} \leq \frac{1}{3}+\frac{1}{2 q}, \frac{15}{2}<q \leq \infty$ and

$$
j_{3} \in L^{p^{\prime}}\left(0, T ; L^{q^{\prime}}\left(R^{3}\right)\right)
$$

with $\frac{2}{p^{\prime}}+\frac{3}{q^{\prime}} \leq 2, \frac{13}{2}<q^{\prime} \leq \infty$, then the weak solution $(u, b)$ is regular, where $j_{3}$ is the third component of $\nabla \times b=\left(j_{1}, j_{2}, j_{3}\right)$. Later Zhang [16] refined the result of Yamazaki's. He proved that, if

$$
u_{3} \in L^{p}\left(0, T ; L^{q}\left(R^{3}\right)\right)
$$

with $\frac{2}{p}+\frac{3}{q} \leq \frac{4}{9}+\frac{1}{3 q}, \frac{15}{2}<q \leq \infty$ and

$$
j_{3} \in L^{p^{\prime}}\left(0, T ; L^{q^{\prime}}\left(R^{3}\right)\right)
$$

with $\frac{2}{p^{\prime}}+\frac{3}{q^{\prime}} \leq 2, \frac{3}{2}<q^{\prime} \leq \infty$, then the weak solution $(u, b)$ is regular.

We further assume that $\omega=\chi=0$, the system (1.1) is usually named MHD equations without magnetic diffusion. In order to present our motivation, we list some information on regularity criteria for 2D-MHD equations without magnetic diffusion. In 2011, Zhou and Fan [18] proved if $\nabla b \in L^{1}\left(0, T ; \mathrm{BMO}\left(R^{2}\right)\right)$, then the local strong solution $(u, b)$ is regular. Gala, Ragusa and Ye [6] improved Zhou and Fan's result. They showed that, if $\nabla b \in L^{\frac{p}{p-1}}\left(0, T ; \dot{M}_{p, q}\left(R^{2}\right)\right)$ with $p \geq q>1$, the local strong solution $(u, b)$ to the MHD equations with magnetic diffusion is regular. Motivated by $[2,15]$, and [6], we will investigate 
the regularity criterion on the weak solution to the magneto-micropolar flows involving one velocity component and the gradient of magnetic field satisfying (1.3) and (1.4). Our result is stated as follows.

Theorem 1.1 Let $\left(u_{0}, b_{0}\right) \in H^{1}\left(R^{3}\right)$ and $\omega_{0} \in H^{1}\left(R^{3}\right)$, with the initial data $\nabla \cdot u_{0}=\nabla \cdot b_{0}=$ 0 . Assume that $(u, \omega, b)$ be the weak solution to the equations (1.1) defined on $[0, T)$ for some $0<T<\infty$. If $(u, b)$ satisfies

$$
u_{3} \in L^{\beta}\left(0, T ; L^{\alpha}\left(R^{3}\right)\right), \quad \frac{2}{\beta}+\frac{3}{\alpha} \leq \frac{3}{4}+\frac{1}{2 \alpha}, \quad \alpha>\frac{10}{3},
$$

and

$$
\nabla b \in L^{\frac{4 p}{3(p-2)}}\left(0, T ; \dot{M}_{p, q}\left(R^{3}\right)\right), \quad 1<q \leq p<\infty, p \geq 3
$$

then the solution $(u, \omega, b)$ to $(1.1)$ can be smoothly extended beyond $t=T$.

Remark 1.1 To the best of our knowledge, this is the first regularity criterion result is concerned with weak solution to the 3D incompressible MHD equations without magnetic diffusion in Morrey Campanato space. The worst difficulty is to handle the nonlinear term $\int_{R^{3}} u \cdot \nabla u \cdot \Delta u d x$. For the two dimension case, due to $\int_{R^{2}} u \cdot \nabla u \cdot \Delta u=0$, the condition $\nabla b \in L^{\frac{2 p}{p-2}}\left(0, T ; \dot{M}_{p, q}\left(R^{2}\right)\right)$ is sufficient (see [6]). Compared with the result in [2], due to the magneto-micropolor fluid flows discussed in Theorem 1.1 there is lack of magnetic diffusion, it increases the difficulties of dealing with the nonlinear terms in $H^{1}$-energy estimates, especially for the term $\int_{R^{3}} u \cdot \nabla w \cdot \Delta w d x$. Fortunately, the $L^{3}$-energy estimate for $\omega$ helps us to overcome these problems.

When $\omega=0, \chi=0$, the magneto-micropolar equations (1.1) become the classical MHD equations without magnetic diffusion. Theorem 1.1 converts into the following corollary.

Corollary 1.1 Let $\left(u_{0}, b_{0}\right) \in H^{1}\left(R^{3}\right)$ with the initial data $\nabla \cdot u_{0}=\nabla \cdot b_{0}=0$. Assume that $(u, b)$ be the weak solution to the incompressible MHD equations defined on $[0, T)$ for some $0<T<\infty$. If $(u, b)$ satisfies

$$
u_{3} \in L^{\beta}\left(0, T ; L^{\alpha}\left(R^{3}\right)\right), \quad \frac{2}{\beta}+\frac{3}{\alpha} \leq \frac{3}{4}+\frac{1}{2 \alpha}, \quad \alpha>\frac{10}{3},
$$

and

$$
\nabla b \in L^{\frac{4 p}{3(p-2)}}\left(0, T ; \dot{M}_{p, q}\left(R^{3}\right)\right), \quad 1<q \leq p<\infty, p \geq 3
$$

then the local strong solution $(u, b)$ can be smoothly extended beyond $t=T$.

Remark 1.2 Comparing with the results either in [15] or in [16], though the MHD equations discussed in Corollary 1.1 show lack of magnetic diffusion and the spatial space of magnetic field is enlightened, it is hard to say our results have refined the one in [15] or that in [16]. 
The difficulties and strategy are listed as follows:

- The first big tiger is to estimate the nonlinear term $\int_{R^{3}} u \cdot \nabla u \cdot \Delta u d x$. Since $\int_{R^{3}} u \cdot \nabla u$. $\Delta u d x \neq 0$, it is impossible to handle it as in the second dimension. We have to present the horizontal energy estimate $\left\|\nabla_{h} u\right\|_{L^{2}},\left\|\nabla_{h} b\right\|_{L^{2}}$.

- The second thorn is the nonlinear term $\int_{R^{3}} u \cdot \nabla w \cdot \Delta w d x$. Integrating by part gives $\int_{R^{3}} \Delta u \cdot \nabla \omega \cdot \omega d x$ and $\int_{R^{3}} \nabla u \cdot \nabla^{2} \omega \cdot \omega d x$. As usual, if we use the Hölder inequality and the Young inequality, we get

$$
\begin{aligned}
\left|\int_{R^{3}} u \cdot \nabla \omega \cdot \Delta \omega d x\right| & \leq\|u\|_{L^{6}}\|\nabla \omega\|_{L^{3}}\|\Delta \omega\|_{L^{2}} \leq\|\nabla u\|_{L^{2}}\|\nabla \omega\|_{L^{2}}^{\frac{1}{2}}\|\Delta \omega\|_{L^{2}}^{\frac{3}{2}} \\
& \leq \frac{1}{4}\|\Delta \omega\|_{L^{2}}^{2}+C\|\nabla u\|_{L^{2}}^{4}\|\nabla \omega\|_{L^{2}}^{2}
\end{aligned}
$$

which does not work. Thanks to the $L^{3}$-norm estimate of $\omega$ and some suitable interpolating inequality, which can be found in Sect. 3, we can overcome these difficulties. More precisely, these methods helps us to handle $\int_{\mathbf{R}^{3}} \Delta u \cdot \nabla \omega \cdot \omega d x$ and $\int_{\mathbf{R}^{3}} \nabla u \cdot \nabla^{2} \omega \cdot \omega d x$ successfully.

The rest of this paper is organized as follows. The definition of some functional spaces and some useful lemmas are presented in Sect. 2. The $L^{3}$-norm of $\omega$ is given in Sect. 3. The proof of Theorem 1.1 is presented in Sect. 4.

\section{Preliminaries and some basic facts}

In this section, we will present some information on the Morrey space and introduce the definition of a weak solution to the magneto-micropolar equation (1.1).

Definition 2.1 (see [10]) For $1<q \leq p \leq \infty$, the homogeneous Morrey space is presented as follows:

$$
\dot{M}_{p, q}=\left\{f \in L_{l o c}^{q}\left(R^{3}\right):\|f\|_{\dot{M}_{p, q}\left(R^{3}\right)}=\sup _{x \in R^{3} R>0} \sup ^{\frac{3}{p}-\frac{3}{q}}\|f\|_{L^{q}(B(x, R))}<\infty\right\},
$$

where $B(x, R)$ denotes the closed ball in $R^{3}$ with center $\mathrm{x}$ and radius $\mathrm{R}$.

Definition 2.2 (see [10]) Let $1<p^{\prime} \leq q^{\prime}<\infty$, we address the homogeneous space $Z_{p^{\prime}, q^{\prime}}$ defined by

$$
\begin{aligned}
Z_{p^{\prime}, q^{\prime}}= & \left\{f \in L^{p^{\prime}} \mid f=\sum_{k \in N} g_{k}, \quad \text { where }\left(g_{k}\right) \subset L_{\text {comp }}^{q^{\prime}}\left(R^{3}\right),\right. \\
& \left.\sum_{k \in N} d_{k}^{3\left(\frac{1}{p^{\prime}}-\frac{1}{q^{\prime}}\right)}\left\|g_{k}\right\|_{L^{q^{\prime}}}<\infty, \quad \text { where } \forall k, d_{k}=\operatorname{diam}\left(\operatorname{supp} g_{k}\right)<\infty\right\} .
\end{aligned}
$$

The following lemma plays a crucial role in proving the regularity criterion for the magneto-micropolar fluid flows (1.1).

Lemma 2.1 (see [10]) (1) Let $1<p^{\prime} \leq q^{\prime}<\infty$ and $p$, $q$ such that $\frac{1}{p}+\frac{1}{p^{\prime}}=1, \frac{1}{q}+\frac{1}{q^{\prime}}=1$, then $\dot{M}_{p, q}$ is the dual space of $Z_{p^{\prime}, q^{\prime}}$. 
(2) Let $1<p^{\prime} \leq q^{\prime}<2, \frac{1}{p}+\frac{1}{p^{\prime}}=1, \frac{1}{q}+\frac{1}{q^{\prime}}=1$ and $r=\frac{3}{p}$, there exists $C>0$ such that $\forall f \in$ $L^{2}\left(R^{3}\right)$ and $\forall g \in \dot{H}^{r}\left(R^{3}\right), h \in \dot{M}_{p, q}\left(R^{3}\right)$ satisfies

$$
\int_{R^{3}}|f(x) g(x) h(x)| d x \leq C\|h\|_{\dot{M}_{p, q}}\|f g\|_{Z_{p^{\prime}, q^{\prime}}} \leq C\|h\|_{\dot{M}_{p, q}}\|f\|_{L^{2}}\|g\|_{\dot{H}^{r}} .
$$

The Sobolev-Ladyzhenskaya inequality in the whole space $R^{3}$ reads as follows.

Lemma 2.2 (see [1]) There exists a constant $C>0$ such that

$$
\|\phi\|_{L^{p}} \leq C\|\phi\|_{L^{2}}^{\frac{6-p}{2 p}}\left\|\partial_{1} \phi\right\|_{L^{2}}^{\frac{p-2}{2 p}}\left\|\partial_{2} \phi\right\|_{L^{2}}^{\frac{p-2}{2 p}}\left\|\partial_{3} \phi\right\|_{L^{2}}^{\frac{p-2}{2 p}}
$$

for every $\phi \in H^{1}\left(R^{3}\right)$ and every $p \in[2,6]$, where $C$ is a constant depending only on $p$.

The definition of a weak solution to the magnetic micropolar equation is provided in the following.

Definition 2.3 Let $\left(u_{0}, b_{0}\right) \in L_{\sigma}^{2}\left(R^{3}\right), \omega \in L^{2}\left(R^{3}\right)$ and $T>0$. A measurable function $(u, b, \omega)$ is said to be a weak solution to $(1.1)$ on $(0, T)$ if

(i) $(u, b) \in L^{\infty}\left(0, T ; L_{\sigma}^{2}\left(R^{3}\right)\right) \cap L^{2}\left(0, T ; H^{1}\left(R^{3}\right)\right)$, and $\omega \in L^{\infty}\left(0, T ; L^{2}\left(R^{3}\right)\right) \cap L^{2}\left(0, T ; H^{1}\left(R^{3}\right)\right)$;

(ii) for every $\phi, \varphi \in H^{1}\left(0, T ; H_{\sigma}^{1}\left(R^{3}\right)\right)$ and $\psi \in H^{1}\left(0, T ; H^{1}\left(R^{3}\right)\right)$ with $\phi(T)=\varphi(T)=$ $\psi(T)=0$,

$$
\begin{aligned}
& \int_{0}^{T}\left\langle-u, \partial_{t} \phi\right\rangle+\langle u \cdot \nabla u, \phi\rangle+(\mu+\chi)\langle\nabla u, \nabla \phi\rangle d t \\
& \quad-\int_{0}^{T}\langle b \cdot \nabla b, \phi\rangle+\chi\langle\nabla \times \omega, \phi\rangle d t=-\left\langle u_{0}, \phi_{0}\right\rangle, \\
& \int_{0}^{T}\left\langle-\omega, \partial_{t} \varphi\right\rangle+\gamma\langle\omega, \nabla \varphi\rangle+\kappa\langle\nabla \cdot u, \nabla \cdot \varphi\rangle d t \\
& \quad+\int_{0}^{T}\langle u \cdot \nabla \omega, \varphi\rangle+2 \chi\langle\omega, \varphi\rangle-2 \chi\langle\nabla \times u, \varphi\rangle d t=-\left\langle\omega_{0}, \phi_{0}\right\rangle,
\end{aligned}
$$

and

$$
\int_{0}^{T}\left\langle-b, \partial_{t} \psi\right\rangle+\langle u \cdot \nabla b, \psi\rangle-\langle b \cdot \nabla u, \psi\rangle d t=-\left\langle b_{0}, \psi_{0}\right\rangle
$$

where $L_{\sigma}^{2}=\left\{u \mid u \in L^{2}, \nabla \cdot u=0\right\}$.

\section{Some useful lemmas}

The following $L^{3}$-energy estimate is needed to prove our result.

Lemma 3.1 Let $(u, \omega, b)$ be the weak solution to the magneto-micropolar equation (1.1). Then

$$
\|\omega\|_{L^{3}}^{3}+\int_{0}^{t}\left\|\nabla|\omega|^{\frac{3}{2}}\right\|_{L^{2}}^{2} d \tau \leq C\left(\left\|\omega_{0}\right\|_{L^{3}},\left\|\omega_{0}\right\|_{L^{2}},\left\|u_{0}\right\|_{L^{2}},\left\|b_{0}\right\|_{L^{2}}, T\right),
$$

for any $t \in[0, T)$. 
Proof Multiplying $(1.1)_{2}$ by $|\omega| \omega$ and integrating over $R^{3}$, we have

$$
\begin{aligned}
& \frac{1}{3} \frac{d}{d t}\|\omega\|_{L^{3}}^{3}+2\|\omega\|_{L^{3}}^{3}+\frac{4}{9}\left\|\nabla|\omega|^{\frac{3}{2}}\right\|_{L^{2}}^{2}+\frac{1}{2}\left\||\omega|^{\frac{1}{2}} \nabla \omega\right\|_{L^{2}}^{2}+\frac{1}{2}\left\||\omega|^{\frac{1}{2}} \nabla \cdot \omega\right\|_{L^{2}}^{2} \\
& \quad \leq\left|\int_{R^{3}} \nabla \times u \cdot\right| \omega|\omega d x|
\end{aligned}
$$

where we have used

$$
\begin{aligned}
\int_{R^{3}}(\nabla \cdot \omega) \nabla \cdot(|\omega| \omega) d x & =\int_{R^{3}} \nabla \cdot \omega(|\omega| \nabla \cdot \omega+\omega \nabla|\omega|) d x \\
& =\left\||\omega|^{\frac{1}{2}} \nabla \cdot \omega\right\|_{L^{2}}^{2}+\int_{R^{3}} \nabla \cdot \omega \cdot \omega \cdot \nabla|\omega| d x \\
& \geq \frac{1}{2}\left\||\omega|^{\frac{1}{2}} \nabla \cdot \omega\right\|_{L^{2}}^{2}-\frac{1}{2}\left\||\omega|^{\frac{1}{2}} \nabla \omega\right\|_{L^{2}}^{2}
\end{aligned}
$$

To estimate the nonlinear term on the right hand side, integrating by parts and using the Young inequality and (4.1), we obtain

$$
\begin{aligned}
\int_{R^{3}} \nabla \times u \cdot|\omega| \omega d x & \leq \int_{R^{3}}|u||\omega||\nabla| \omega|| d x+\int_{R^{3}}|u||\omega||\nabla \times \omega| d x \\
& \leq C \int_{R^{3}}|u||\omega||\nabla \omega| d x \\
& \leq C\|u\|_{L^{3}}\left\||\omega|^{\frac{1}{2}}\right\|_{L^{6}}\left\||\omega|^{\frac{1}{2}} \nabla \omega\right\|_{L^{2}} \\
& \leq \frac{1}{4}\left\||\omega|^{\frac{1}{2}} \nabla \omega\right\|_{L^{2}}^{2}+\frac{1}{2}\|\omega\|_{L^{3}}^{3}+C\|\nabla u\|_{L^{2}}^{\frac{3}{2}} \\
& \leq \frac{1}{4}\left\||\omega|^{\frac{1}{2}} \nabla \omega\right\|_{L^{2}}^{2}+\frac{1}{2}\|\omega\|_{L^{3}}^{3}+C\left(\|\nabla u\|_{L^{2}}^{2}+1\right) .
\end{aligned}
$$

Substituting the above inequality into (3.2) gives

$$
\begin{aligned}
& \frac{d}{d t}\|\omega\|_{L^{3}}^{3}+\|\omega\|_{L^{3}}^{3}+\left\|\nabla|\omega|^{\frac{3}{2}}\right\|_{L^{2}}^{2}+\frac{3}{4}\left\||\omega|^{\frac{1}{2}} \nabla \omega\right\|_{L^{2}}^{2}+\left\||\omega|^{\frac{1}{2}} \nabla \cdot \omega\right\|_{L^{2}}^{2} \\
& \quad \leq C\left(\|\nabla u\|_{L^{2}}^{2}+1\right) .
\end{aligned}
$$

Integrating on $[0, t)$ and using $(4.1)$ yield

$$
\begin{aligned}
\|\omega\|_{L^{3}}^{3}+\int_{0}^{t}\left\|\nabla|\omega|^{\frac{3}{2}}\right\|_{L^{2}}^{2} d \tau & \leq\left\|\omega_{0}\right\|_{L^{3}}^{3}+C \int_{0}^{t}\left(\|\nabla u\|_{L^{2}}^{2}+1\right) d \tau \\
& \leq C\left(\left\|\omega_{0}\right\|_{L^{3}},\left\|\omega_{0}\right\|_{L^{2}},\left\|u_{0}\right\|_{L^{2}},\left\|b_{0}\right\|_{L^{2}}, T\right) .
\end{aligned}
$$

\section{Proof of Theorem 1.1}

In this section, we shall give the proof of Theorem 1.1. We will assume that $\mu=\chi=\gamma=$ $\kappa=1$ throughout this paper.

Let $\left[0, T^{*}\right)$ be the maximal time interval for the existence of the local smooth solution. If $T^{*} \geq T$, the conclusion is obviously valid, but for $T^{*}<T$, we would show that

$$
\lim \sup _{t \rightarrow T^{*}}\left(\|\nabla u(\cdot, t)\|_{L^{2}}^{2}+\|\nabla \omega(\cdot, t)\|_{L^{2}}^{2}+\|\nabla b(\cdot, t)\|_{L^{2}}^{2}\right) \leq C
$$


under the assumption of (1.3) and (1.4). Hence, according to the definition of $T^{*}$, this leads to a contradiction.

Step 1: $L^{2}$-energy estimate A standard energy method says

$$
\begin{aligned}
& \|(u(t), \omega(t), b(t))\|_{L^{2}}^{2}+2 \int_{0}^{t}\|\nabla u\|_{L^{2}}^{2} d \tau+2 \int_{0}^{t}\|\nabla \omega\|_{L^{2}}^{2} d \tau \\
& \quad+2 \int_{0}^{t}\|\nabla \cdot \omega\|_{L^{2}}^{2} d \tau+2 \int_{0}^{t}\|\omega\|_{L^{2}}^{2} d \tau \leq\left\|\left(u_{0}, \omega_{0}, b_{0}\right)\right\|_{L^{2}}^{2} .
\end{aligned}
$$

Step 2: $H^{1}$-Horizontal energy estimate

We first establish the horizontal gradient of the velocity $u$ and magnetic field $b$. Taking $\nabla_{h}$ on both sides of Eqs. $(1.1)_{1}$ and $(1.1)_{3}$, multiplying by $\nabla_{h} u$ and $\nabla_{h} b$, respectively, and integrating over $\mathbf{R}^{3}$, we get

$$
\begin{aligned}
\frac{1}{2} \frac{d}{d t}( & \left.\left\|\nabla_{h} u\right\|_{L^{2}}^{2}+\left\|\nabla_{h} b\right\|_{L^{2}}^{2}\right)+2\left\|\nabla_{h} \nabla u\right\|_{L^{2}}^{2} \\
= & -\int_{R^{3}} \nabla_{h}(u \cdot \nabla u) \cdot \nabla_{h} u d x \\
& +\int_{R^{3}}\left(\nabla_{h}(b \cdot \nabla b) \cdot \nabla_{h} u+\nabla_{h}(b \cdot \nabla u) \cdot \nabla_{h} b-\nabla_{h}(u \cdot \nabla b) \cdot \nabla_{h} b\right) d x \\
& +\int_{R^{3}} \nabla_{h}(\nabla \times \omega) \cdot \nabla_{h} u d x \\
:= & A_{1}+A_{2}+A_{3} .
\end{aligned}
$$

We start to estimate $A_{2}$. From $(1.1)_{4}$ and Lemma 2.1 and the fact $\nabla \cdot u=\nabla \cdot b=0$, we know that $(p \geq 3)$

$$
\begin{aligned}
A_{2}= & \int_{R^{3}} \sum_{k=1}^{2} \partial_{k}(b \cdot \nabla b) \partial_{k} u d x+\int_{R^{3}} \sum_{k=1}^{2} \partial_{k}(b \cdot \nabla u) \partial_{k} b d x \\
& -\int_{R^{3}} \sum_{k=1}^{2} \partial_{k}(u \cdot \nabla b) \partial_{k} b d x \\
= & \int_{R^{3}} \sum_{k=1}^{2}\left(\partial_{k} b \cdot \nabla b \partial_{k} u+\partial_{k} b \cdot \nabla u \partial_{k} b\right) d x-\int_{\mathbf{R}^{3}} \sum_{k=1}^{2} \partial_{k} u \cdot \nabla b \partial_{k} b d x \\
\leq & C \int_{R^{3}}|\nabla b\|\nabla u\| \nabla b| d x \leq C\|\nabla b\|_{\dot{M}_{p, q}}\|\nabla b\|_{L^{2}}\|\nabla u\|_{\dot{H}^{\frac{3}{p}}} \\
\leq & C\|\nabla b\|_{\dot{M}_{p, q}}\|\nabla b\|_{L^{2}}\|\nabla u\|_{L^{2}}^{1-\frac{3}{p}}\|\Delta u\|_{L^{2}}^{\frac{3}{p}} .
\end{aligned}
$$

Thanks to the Hölder and Young inequalities, one deduces

$$
\begin{aligned}
A_{3} & =\int_{R^{3}} \sum_{k=1}^{2} \partial_{k}(\nabla \times \omega) \cdot \partial_{k} u d x-\int_{R^{3}} \sum_{k=1}^{2} \nabla \times \omega \cdot \partial_{k} \partial_{k} u d x \\
& \leq C\|\nabla \times \omega\|_{L^{2}}\left\|\nabla_{h} \nabla u\right\|_{L^{2}} \\
& \leq \frac{1}{2}\left\|\nabla_{h} \nabla u\right\|_{L^{2}}^{2}+C\|\nabla \omega\|_{L^{2}}^{2} .
\end{aligned}
$$


Now it is time to deal with the first term $A_{1}$. Integrating by parts, we get

$$
\begin{aligned}
A_{1}= & \int_{R^{3}} u \cdot \nabla u \cdot \Delta_{h} u d x \\
= & \int_{R^{3}} \sum_{k=1}^{2} \sum_{i, j=1}^{2} u_{i} \partial_{i} u_{j} \partial_{k} \partial_{k} u_{j} d x+\int_{\mathbf{R}^{3}} \sum_{k=1}^{2} \sum_{i=1}^{2} u_{i} \partial_{i} u_{3} \partial_{k} \partial_{k} u_{3} d x \\
& +\int_{R^{3}} \sum_{k=1}^{2} \sum_{j=1}^{3} u_{3} \partial_{3} u_{j} \partial_{k} \partial_{k} u_{j} d x \\
:= & A_{11}+A_{12}+A_{13} .
\end{aligned}
$$

Using integration by parts again and applying the fact that $\operatorname{div} u=0$, it yields

$$
\begin{aligned}
A_{11}= & -\int_{R^{3}} \sum_{k=1}^{2} \sum_{i, j=1}^{2}\left(\partial_{k} u_{i} \partial_{i} u_{j} \partial_{k} u_{j}+u_{i} \partial_{i} \partial_{k} u_{j} \partial_{k} u_{j}\right) d x \\
= & \int_{R^{3}} \sum_{k=1}^{2} \sum_{i, j=1}^{2} \partial_{k} u_{i} \partial_{i} u_{j} \partial_{k} u_{j} d x+\frac{1}{2} \int_{R^{3}} \sum_{k=1}^{2} \sum_{i, j=1}^{2} \partial_{i} u_{i} \partial_{k} u_{j} \partial_{k} u_{j} d x \\
= & \int_{R^{3}} \sum_{k=1}^{2} \sum_{i, j=1}^{2} \partial_{k} u_{i} \partial_{i} u_{j} \partial_{k} u_{j} d x-\frac{1}{2} \int_{R^{3}} \sum_{k=1}^{2} \sum_{j=1}^{2} \partial_{3} u_{3} \partial_{k} u_{j} \partial_{k} u_{j} d x \\
= & \int_{R^{3}} \sum_{k=1}^{2} \sum_{i, j=1}^{2} \partial_{k} u_{i} \partial_{i} u_{j} \partial_{k} u_{j} d x-\frac{1}{2} \int_{R^{3}} \sum_{k=1}^{2} \sum_{j=1}^{2} \partial_{3} u_{3} \partial_{k} u_{j} \partial_{k} u_{j} d x \\
= & -\int_{R^{3}} \partial_{3} u_{3}\left(\left(\partial_{1} u_{2}\right)^{2}+\partial_{2} u_{1} \partial_{1} u_{2}+\left(\partial_{2} u_{1}\right)^{2}+\left(\partial_{1} u_{1}\right)^{2}-\partial_{1} u_{1} \partial_{2} u_{2}+\left(\partial_{2} u_{2}\right)^{2}\right) d x \\
& -\frac{1}{2} \int_{R^{3}} \sum_{k=1}^{2} \sum_{j=1}^{2} \partial_{3} u_{3} \partial_{k} u_{j} \partial_{k} u_{j}
\end{aligned}
$$

and

$$
\begin{aligned}
A_{12}= & -\int_{R^{3}} \sum_{k=1}^{2} \sum_{i=1}^{2}\left(\partial_{k} u_{i} \partial_{i} u_{3} \partial_{k} u_{3}+u_{i} \partial_{i} \partial_{k} u_{3} \partial_{k} u_{3}\right) d x \\
= & \int_{R^{3}} \sum_{k=1}^{2} \sum_{i=1}^{2}\left(\partial_{k} \partial_{i} u_{i} u_{3} \partial_{k} u_{3}+\partial_{k} u_{i} u_{3} \partial_{k} \partial_{i} u_{3}\right) d x \\
& +\frac{1}{2} \int_{R^{3}} \sum_{k=1}^{2} \sum_{i=1}^{2} \partial_{i} u_{i} \partial_{k} u_{3} \partial_{k} u_{3} d x \\
= & \int_{R^{3}} \sum_{k=1}^{2} \sum_{i=1}^{2}\left(\partial_{k} \partial_{i} u_{i} u_{3} \partial_{k} u_{3}+\partial_{k} u_{i} u_{3} \partial_{k} \partial_{i} u_{3}\right) d x \\
& -\frac{1}{2} \int_{R^{3}} \sum_{k=1}^{2} \partial_{3} u_{3} \partial_{k} u_{3} \partial_{k} u_{3} d x \\
= & \int_{R^{3}} \sum_{k=1}^{2} \sum_{i=1}^{2}\left(\partial_{k} \partial_{i} u_{i} u_{3} \partial_{k} u_{3}+\partial_{k} u_{i} u_{3} \partial_{k} \partial_{i} u_{3}\right) d x
\end{aligned}
$$




$$
+\int_{R^{3}} \sum_{k=1}^{2} u_{3} \partial_{3} \partial_{k} u_{3} \partial_{k} u_{3} d x
$$

Substituting (4.6) and (4.7) into (4.5) yields

$$
A_{1} \leq C \int_{\mathbf{R}^{3}}\left|u_{3}\right||\nabla u|\left|\nabla_{h} \nabla u\right| d x
$$

Thanks to the Hölder inequality, (2.1) and the Young inequality, we obtain for $\alpha>3$

$$
\begin{aligned}
A_{1} & \leq C\left\|u_{3}\right\|_{L^{\alpha}}\left\|\nabla_{h} \nabla u\right\|_{L^{2}}\|\nabla u\|_{L^{\frac{2 \alpha}{\alpha-2}}} \\
& \leq C\left\|u_{3}\right\|_{L^{\alpha}}\left\|\nabla_{h} \nabla u\right\|_{L^{2}}\|\nabla u\|_{L^{2}}^{1-\frac{3}{\alpha}}\|\nabla u\|_{L^{6}}^{\frac{3}{\alpha}} \\
& \leq C\left\|u_{3}\right\|_{L^{\alpha}}\left\|\nabla_{h} \nabla u\right\|_{L^{2}}\|\nabla u\|_{L^{2}}^{1-\frac{3}{\alpha}}\left\|\nabla_{h} \nabla u\right\|_{L^{2}}^{\frac{2}{\alpha}}\left\|\partial_{3} \nabla u\right\|_{L^{2}}^{\frac{1}{\alpha}} \\
& \leq \frac{1}{2}\left\|\nabla_{h} \nabla u\right\|_{L^{2}}^{2}+C\left\|u_{3}\right\|_{L^{\alpha}}^{\frac{2 \alpha}{\alpha-2}}\|\nabla u\|_{L^{2}}^{\frac{2(\alpha-3)}{\alpha-2}}\|\Delta u\|_{L^{2}}^{\frac{2}{\alpha-2}},
\end{aligned}
$$

which, along with (4.3), (4.4) and (4.2), gives

$$
\begin{aligned}
& \frac{d}{d t}\left(\left\|\nabla_{h} u\right\|_{L^{2}}^{2}+\left\|\nabla_{h} b\right\|_{L^{2}}^{2}\right)+2\left\|\nabla_{h} \nabla u\right\|_{L^{2}}^{2} \\
& \leq C\left\|u_{3}\right\|_{L^{\alpha}}^{\frac{2 \alpha}{\alpha-2}}\|\nabla u\|_{L^{2}}^{\frac{2(\alpha-3)}{\alpha-2}}\|\Delta u\|_{L^{2}}^{\frac{2}{\alpha-2}}+C\|\nabla \omega\|_{L^{2}}^{2} \\
& \quad+C\|\nabla b\|_{\dot{M}_{p, q}}\|\nabla b\|_{L^{2}}\|\nabla u\|_{L^{2}}^{1-\frac{3}{p}}\|\Delta u\|_{L^{2}}^{\frac{3}{p}}
\end{aligned}
$$

Integrating over $[0, t)$ and using (4.1), one can verify

$$
\begin{aligned}
& \sup _{0 \leq \tau \leq t}\left(\left\|\nabla_{h} u\right\|_{L^{2}}^{2}+\left\|\nabla_{h} b\right\|_{L^{2}}^{2}\right)+2 \int_{0}^{t}\left\|\nabla_{h} \nabla u\right\|_{L^{2}}^{2} d \tau \\
& \leq C\left(\left\|\nabla_{h} u_{0}\right\|_{L^{2}}^{2}+\left\|\nabla_{h} b_{0}\right\|_{L^{2}}^{2}+1\right)+C \int_{0}^{t}\left\|u_{3}\right\|_{L^{\alpha}}^{\frac{2 \alpha}{\alpha-2}}\|\nabla u\|_{L^{2}}^{\frac{2(\alpha-3)}{\alpha-2}}\|\Delta u\|_{L^{2}}^{\frac{2}{\alpha-2}} d \tau \\
& \quad+C \int_{0}^{t}\|\nabla b\|_{\dot{M}_{p, q}}\|\nabla b\|_{L^{2}}\|\nabla u\|_{L^{2}}^{1-\frac{3}{p}}\|\Delta u\|_{L^{2}}^{\frac{3}{p}} d \tau .
\end{aligned}
$$

\section{Step 3: $H^{1}$-full energy estimate}

Multiplying $(1.1)_{1},(1.1)_{2}$ and $(1.1)_{3}$ by $-\Delta u,-\Delta \omega$ and $-\Delta b$, respectively, and integrating over $\mathbf{R}^{3}$, then adding them we obtain

$$
\begin{aligned}
\frac{1}{2} & \frac{d}{d t}\left(\|\nabla u\|_{L^{2}}^{2}+\|\nabla \omega\|_{L^{2}}^{2}+\|\nabla b\|_{L^{2}}^{2}\right)+2\|\Delta u\|_{L^{2}}^{2}+\|\Delta \omega\|_{L^{2}}^{2}+\|\nabla \nabla \cdot \omega\|_{L^{2}}^{2} \\
= & \int_{R^{3}} u \cdot \nabla u \cdot \Delta u d x-\int_{\mathbf{R}^{3}} b \cdot \nabla b \cdot \Delta u d x+\int_{R^{3}} u \cdot \nabla \omega \cdot \Delta \omega d x \\
& -2 \int_{R^{3}} \nabla \times u \cdot \Delta \omega d x+\int_{R^{3}} u \cdot \nabla b \cdot \Delta b d x-\int_{R^{3}} b \cdot \nabla u \cdot \Delta b d x \\
:= & B_{1}+B_{2}+B_{3}+B_{4}+B_{5}+B_{6}
\end{aligned}
$$


where we have used the inequalities

$$
\begin{aligned}
\int_{R^{3}}(-\nabla \nabla \cdot \omega)(-\Delta \omega) d x & =\sum_{i, j, k=1}^{3} \int_{R^{3}} \partial_{i} \partial_{j} \omega_{j} \partial_{k} \partial_{k} \omega_{i} d x \\
& =\sum_{i, j, k=1}^{3} \int_{R^{3}} \partial_{k} \partial_{j} \omega_{j} \partial_{k} \partial_{i} \omega_{i} d x \\
& =\|\nabla \nabla \cdot \omega\|_{L^{2}}^{2}
\end{aligned}
$$

and

$$
\int_{R^{3}} \nabla \times \omega \cdot \Delta u d x=\int_{\mathbf{R}^{3}} \Delta \omega \cdot \nabla \times u d x
$$

Now, we estimate $B_{2}, B_{5}$ and $B_{6}$. After applying integration by parts, $\operatorname{div} u=\operatorname{div} b=0$, the Hölder inequality, Lemma 2.1, the Sobolev interpolation inequality and the Young inequality, we have

$$
\begin{aligned}
B_{2} & +B_{5}+B_{6} \\
& =\int_{R^{3}} \nabla b \cdot \nabla b \cdot \nabla u d x-\int_{R^{3}} \nabla u \cdot \nabla b \cdot \nabla b d x+\int_{R^{3}} \nabla b \cdot \nabla u \cdot \nabla b d x \\
& \leq C \int_{R^{3}}|\nabla b||\nabla u \| \nabla b| d x \\
& \leq C\|\nabla b\|_{\dot{M}_{p, q}}\|\nabla b\|_{L^{2}}\|\nabla u\|_{\dot{H}^{\frac{3}{p}}} \\
& \leq C\|\nabla b\|_{\dot{M}_{p, q}}\|\nabla b\|_{L^{2}}\|\nabla u\|_{L^{2}}^{1-\frac{3}{p}}\|\Delta u\|_{L^{2}}^{\frac{3}{p}} \\
& \leq \frac{1}{4}\|\Delta u\|_{L^{2}}^{2}+C\|\nabla b\|_{\dot{M}_{p, q}}^{\frac{2 p}{2 p-3}}\|\nabla b\|_{L^{2}}^{\frac{2 p-3}{2 p-3}}\|\nabla u\|_{L^{2}}^{\frac{2(p-3)}{2 p-3}} \\
& \leq \frac{1}{4}\|\Delta u\|_{L^{2}}^{2}+C\|\nabla b\|_{\dot{M}_{p, q}}^{\frac{2 p}{2 p-3}}\left(\|\nabla b\|_{L^{2}}^{2}+\|\nabla u\|_{L^{2}}^{2} .\right.
\end{aligned}
$$

We can infer from the Hölder and the Young inequalities that

$$
B_{4} \leq 2 \int_{R^{3}} \nabla(\nabla \times u) \cdot \nabla \omega d x \leq \frac{1}{4}\|\Delta u\|_{L^{2}}^{2}+C\|\nabla \omega\|_{L^{2}}^{2} .
$$

Applying integration by parts we obtain

$$
\begin{aligned}
B_{3} & =-\int_{R^{3}} \nabla u \cdot \nabla \omega \cdot \nabla \omega d x \\
& =\int_{R^{3}} \nabla u \cdot \nabla(\nabla \omega) \cdot \omega d x+\int_{R^{3}} \nabla(\nabla u) \cdot \nabla \omega \cdot \omega d x \\
& :=B_{31}+B_{32} .
\end{aligned}
$$


Thanks to the Hölder inequality, the interpolation inequality with $3 \leq \alpha \leq 9$ and Lemma 3.1, we arrive at

$$
\begin{aligned}
B_{31} & \leq \int_{R^{3}}|\nabla u\|\Delta \omega\| \omega| d x \leq\|\Delta \omega\|_{L^{2}}\|\omega\|_{L^{\alpha}}\|\nabla u\|_{L^{\frac{2 \alpha}{\alpha-2}}} \\
& \leq\|\Delta \omega\|_{L^{2}}\|\omega\|_{L^{3}}^{\frac{9-\alpha}{2 \alpha}}\|\omega\|_{L^{9}}^{\frac{3 \alpha-9}{2 \alpha}}\|\nabla u\|_{L^{2}}^{1-\frac{3}{\alpha}}\|\nabla u\|_{L^{6}}^{\frac{3}{\alpha}} \\
& \leq\|\Delta \omega\|_{L^{2}}\left\||\omega|^{\frac{3}{2}}\right\|_{L^{6}}^{1-\frac{3}{\alpha}}\|\nabla u\|_{L^{2}}^{1-\frac{3}{\alpha}}\|\Delta u\|_{L^{2}}^{\frac{3}{\alpha}} \\
& \leq\|\Delta \omega\|_{L^{2}}\left\|\nabla|\omega|^{\frac{3}{2}}\right\|_{L^{2}}^{1-\frac{3}{\alpha}}\|\nabla u\|_{L^{2}}^{1-\frac{3}{\alpha}}\|\Delta u\|_{L^{2}}^{\frac{3}{\alpha}} \\
& \leq \frac{1}{4}\|\Delta \omega\|_{L^{2}}^{2}+C\left\|\nabla|\omega|^{\frac{3}{2}}\right\|_{L^{2}}^{2\left(1-\frac{3}{\alpha}\right)}\|\nabla u\|_{L^{2}}^{2\left(1-\frac{3}{\alpha}\right)}\|\Delta u\|_{L^{2}}^{\frac{6}{\alpha}} \\
& \leq \frac{1}{4}\|\Delta \omega\|_{L^{2}}^{2}+\frac{1}{4}\|\Delta u\|_{L^{2}}^{2}+C\left\|\nabla|\omega|^{\frac{3}{2}}\right\|_{L^{2}}^{2}\|\nabla u\|_{L^{2}}^{2} .
\end{aligned}
$$

Similarly, the term $B_{32}$ can be bounded as follows:

$$
\begin{aligned}
B_{32} & \leq \int_{R^{3}}\left|\Delta u \left\|\nabla \omega \left|\|\omega \mid d x \leq\| \Delta u\left\|_{L^{2}}\right\| \omega\left\|_{L^{\alpha}}\right\| \nabla \omega \|_{L^{\frac{2 \alpha}{\alpha-2}}}\right.\right.\right. \\
& \leq\|\Delta u\|_{L^{2}}\|\omega\|_{L^{3}}^{\frac{9-\alpha}{2 \alpha}}\|\omega\|_{L^{9}}^{\frac{3 \alpha-9}{2 \alpha}}\|\nabla \omega\|_{L^{2}}^{1-\frac{3}{\alpha}}\|\nabla \omega\|_{L^{6}}^{\frac{3}{\alpha}} \\
& \leq\|\Delta u\|_{L^{2}}\left\|\left.\omega\right|^{\frac{3}{2}}\right\|_{L^{6}}^{1-\frac{3}{\alpha}}\|\nabla \omega\|_{L^{2}}^{1-\frac{3}{\alpha}}\|\Delta \omega\|_{L^{2}}^{\frac{3}{\alpha}} \\
& \leq\|\Delta u\|_{L^{2}}\left\|\nabla|\omega|^{\frac{3}{2}}\right\|_{L^{2}}^{1-\frac{3}{\alpha}}\|\nabla \omega\|_{L^{2}}^{1-\frac{3}{\alpha}}\|\Delta \omega\|_{L^{2}}^{\frac{3}{\alpha}} \\
& \leq \frac{1}{4}\|\Delta u\|_{L^{2}}^{2}+C\left\|\nabla|\omega|^{\frac{3}{2}}\right\|_{L^{2}}^{2\left(1-\frac{3}{\alpha}\right)}\|\nabla \omega\|_{L^{2}}^{2\left(1-\frac{3}{\alpha}\right)}\|\Delta \omega\|_{L^{2}}^{\frac{6}{\alpha}} \\
& \leq \frac{1}{4}\|\Delta u\|_{L^{2}}^{2}+\frac{1}{4}\|\Delta \omega\|_{L^{2}}^{2}+C\left\|\nabla|\omega|^{\frac{3}{2}}\right\|_{L^{2}}^{2}\|\nabla \omega\|_{L^{2}}^{2} .
\end{aligned}
$$

For the term $B_{1}$, similar to $A_{1}$, we find that

$$
\begin{aligned}
B_{1}= & \int_{R^{3}} u \cdot \nabla u \cdot \Delta_{h} u d x+\int_{R^{3}} u \cdot \nabla u \cdot \partial_{3} \partial_{3} u d x \\
= & -\int_{R^{3}} \sum_{k=1}^{2} \sum_{i, j=1}^{3} \partial_{k} u_{i} \partial_{i} u_{j} \partial_{k} u_{j} d x-\int_{\mathbf{R}^{3}} \sum_{k=1}^{2} \sum_{i, j=1}^{3} u_{i} \partial_{i} \partial_{k} u_{j} \partial_{k} u_{j} d x \\
& +\int_{R^{3}} \sum_{i, j=1}^{3} u_{i} \partial_{i} u_{j} \partial_{3} \partial_{3} u_{j} d x \\
= & -\int_{R^{3}} \sum_{k=1}^{2} \sum_{i, j=1}^{3} \partial_{k} u_{i} \partial_{i} u_{j} \partial_{k} u_{j} d x-\int_{R^{3}} \sum_{i, j=1}^{3} \partial_{3} u_{i} \partial_{i} u_{j} \partial_{3} u_{j} d x \\
& -\int_{\mathbf{R}^{3}} \sum_{i, j=1}^{3} u_{i} \partial_{i} \partial_{3} u_{j} \partial_{3} u_{j} d x \\
= & -\int_{R^{3}} \sum_{k=1}^{2} \sum_{i, j=1}^{3} \partial_{k} u_{i} \partial_{i} u_{j} \partial_{k} u_{j} d x-\int_{R^{3}} \sum_{j=1}^{3} \sum_{i=1}^{2} \partial_{3} u_{i} \partial_{i} u_{j} \partial_{3} u_{j} d x
\end{aligned}
$$




$$
\begin{aligned}
& -\int_{\mathbf{R}^{3}} \sum_{j=1}^{3} \partial_{3} u_{3} \partial_{3} u_{j} \partial_{3} u_{j} d x \\
\leq & C \int_{R^{3}}\left|\nabla_{h} u\left\|\left.\nabla u\right|^{2} d x \leq C\right\| \nabla_{h} u\left\|_{L^{2}}\right\| \nabla u \|_{L^{4}}^{2}\right. \\
\leq & C\left\|\nabla_{h} u\right\|_{L^{2}}\|\nabla u\|_{L^{2}}^{\frac{1}{2}}\|\nabla u\|_{L^{6}}^{\frac{3}{2}} \\
\leq & C\left\|\nabla_{h} u\right\|_{L^{2}}\|\nabla u\|_{L^{2}}^{\frac{1}{2}}\left\|\partial_{1} \nabla u\right\|_{L^{2}}^{\frac{1}{2}}\left\|\partial_{2} \nabla u\right\|_{L^{2}}^{\frac{1}{2}}\left\|\partial_{3} \nabla u\right\|_{L^{2}}^{\frac{1}{2}} \\
\leq & C\left\|\nabla_{h} u\right\|_{L^{2}}\|\nabla u\|_{L^{2}}^{\frac{1}{2}}\left\|\nabla_{h} \nabla u\right\|_{L^{2}}\|\Delta u\|_{L^{2}}^{\frac{1}{2}} .
\end{aligned}
$$

Combining (4.13), (4.14), (4.16), (4.17) and (4.18), then (4.12) becomes

$$
\begin{gathered}
\frac{d}{d t}\left(\|\nabla u\|_{L^{2}}^{2}+\|\nabla \omega\|_{L^{2}}^{2}+\|\nabla b\|_{L^{2}}^{2}\right)+\|\Delta u\|_{L^{2}}^{2}+\|\Delta \omega\|_{L^{2}}^{2}+\|\nabla \omega\|_{L^{2}}^{2} \\
\leq C\left(\|\nabla b\|_{\dot{M}_{p, q}}^{\frac{2 p}{2 p-3}}+\left\|\nabla|\omega|^{\frac{3}{2}}\right\|_{L^{2}}^{2}\right)\left(\|\nabla u\|_{L^{2}}^{2}+\|\nabla \omega\|_{L^{2}}^{2}+\|\nabla b\|_{L^{2}}^{2}\right) \\
\quad+C\|\nabla \omega\|_{L^{2}}^{2}+C\left\|\nabla_{h} u\right\|_{L^{2}}\|\nabla u\|_{L^{2}}^{\frac{1}{2}}\left\|\nabla_{h} \nabla u\right\|_{L^{2}}\|\Delta u\|_{L^{2}}^{\frac{1}{2}} .
\end{gathered}
$$

Integrating over $[0, t]$ yields

$$
\begin{aligned}
& \left(\|\nabla u\|_{L^{2}}^{2}+\|\nabla \omega\|_{L^{2}}^{2}+\|\nabla b\|_{L^{2}}^{2}\right)+\int_{0}^{t}\|\Delta u\|_{L^{2}}^{2}+\|\Delta \omega\|_{L^{2}}^{2} d \tau \\
& \leq C \int_{0}^{t}\left(\|\nabla b\|_{\dot{M}_{p, q}^{2 p-3}}^{2 p}+\left\|\nabla|\omega|^{\frac{3}{2}}\right\|_{L^{2}}^{2}\right)\left(\|\nabla u\|_{L^{2}}^{2}+\|\nabla \omega\|_{L^{2}}^{2}+\|\nabla b\|_{L^{2}}^{2}\right) d \tau \\
& \quad+C\left(\left\|\nabla u_{0}\right\|_{L^{2}}^{2}+\left\|\nabla \omega_{0}\right\|_{L^{2}}^{2}+\left\|\nabla b_{0}\right\|_{L^{2}}^{2}+1\right)+C G(t),
\end{aligned}
$$

where

$$
G(t)=\int_{0}^{t}\left\|\nabla_{h} u\right\|_{L^{2}}\left\|\nabla_{h} \nabla u\right\|_{L^{2}}\|\nabla u\|_{L^{2}}^{\frac{1}{2}}\|\Delta u\|_{L^{2}}^{\frac{1}{2}} d \tau .
$$

We proceed to estimate $G(t)$. From (4.1) and (4.11), we deduce that

$$
\begin{aligned}
G(t) \leq & \sup _{0 \leq \tau \leq t}\left\|\nabla_{h} u\right\|_{L^{2}}\left(\int_{0}^{t}\left\|\nabla_{h} \nabla u\right\|_{L^{2}}^{2} d \tau\right)^{\frac{1}{2}}\left(\int_{0}^{t}\|\nabla u\|_{L^{2}}^{2}\right)^{\frac{1}{4}}\left(\int_{0}^{t}\|\Delta u\|_{L^{2}}^{2} d \tau\right)^{\frac{1}{4}} \\
\leq & C\left(\sup _{0 \leq \tau \leq t}\left\|\nabla_{h} u\right\|_{L^{2}}^{2}+\int_{0}^{t}\left\|\nabla_{h} \nabla u\right\|_{L^{2}}^{2} d \tau\right)\left(\int_{0}^{t}\|\Delta u\|_{L^{2}}^{2} d \tau\right)^{\frac{1}{4}} \\
\leq & C\left(\left(\left\|\nabla_{h} u_{0}\right\|_{L^{2}}^{2}+\left\|\nabla_{h} b_{0}\right\|_{L^{2}}^{2}+1\right)+\int_{0}^{t}\left\|u_{3}\right\|_{L^{\alpha}}^{\frac{2 \alpha}{\alpha-2}}\|\nabla u\|_{L^{2}}^{\frac{2(\alpha-3)}{\alpha-2}}\|\Delta u\|_{L^{2}}^{\frac{2}{\alpha-2}} d \tau\right. \\
& \left.+\int_{0}^{t}\|\nabla b\|_{\dot{M}_{p, q}}\|\nabla b\|_{L^{2}}\|\nabla u\|_{L^{2}}^{1-\frac{3}{p}}\|\Delta u\|_{L^{2}}^{\frac{3}{p}} d \tau\right)\left(\int_{0}^{t}\|\Delta u\|_{L^{2}}^{2} d \tau\right)^{\frac{1}{4}} \\
\leq & C\left(\left\|\nabla_{h} u_{0}\right\|_{L^{2}}^{\frac{8}{3}}+\left\|\nabla_{h} b_{0}\right\|_{L^{2}}^{\frac{8}{3}}+1\right)+C\left(\int_{0}^{t}\left\|u_{3}\right\|_{L^{\alpha}}^{\frac{2 \alpha}{\alpha-2}}\|\nabla u\|_{L^{2}}^{\frac{2(\alpha-3)}{\alpha-2}}\|\Delta u\|_{L^{2}}^{\frac{2}{\alpha-2}} d \tau\right)^{\frac{4}{3}} \\
& +\left(\int_{0}^{t}\|\nabla b\|_{\dot{M}_{p, q}}\|\nabla b\|_{L^{2}}\|\nabla u\|_{L^{2}}^{1-\frac{3}{p}}\|\Delta u\|_{L^{2}}^{\frac{3}{p}} d \tau\right)^{\frac{4}{3}}+\frac{1}{4} \int_{0}^{t}\|\Delta u\|_{L^{2}}^{2} d \tau
\end{aligned}
$$




$$
\begin{aligned}
& \leq C\left(\left\|\nabla_{h} u_{0}\right\|_{L^{2}}^{\frac{8}{3}}+\left\|\nabla_{h} b_{0}\right\|_{L^{2}}^{\frac{8}{3}}+1\right)+\frac{1}{4} \int_{0}^{t}\|\Delta u\|_{L^{2}}^{2} d \tau \\
& +\left(\int_{0}^{t}\left\|u_{3}\right\|_{L^{\alpha}}^{\frac{2 \alpha}{\alpha-3}}\|\nabla u\|_{L^{2}}^{2} d \tau\right)^{\frac{4(\alpha-3)}{3(\alpha-2)}}\left(\int_{0}^{t}\|\Delta u\|_{L^{2}}^{2} d \tau\right)^{\frac{4}{3(\alpha-2)}} \\
& +C\left(\int_{0}^{t}\|\nabla b\|_{\dot{M}_{p, q}}^{\frac{2 p}{2 p-3}}\|\nabla b\|_{L^{2}}^{\frac{2 p}{2 p-3}}\|\nabla u\|_{L^{2}}^{\frac{2(p-3)}{2 p-3}} d \tau\right)^{\frac{2(2 p-3)}{3 p}}\left(\int_{0}^{t}\|\Delta u\|_{L^{2}}^{2} d \tau\right)^{\frac{2}{p}} \\
& \leq C\left(\left\|\nabla_{h} u_{0}\right\|_{L^{2}}^{\frac{8}{3}}+\left\|\nabla_{h} b_{0}\right\|_{L^{2}}^{\frac{8}{3}}+1\right)+\frac{3}{4} \int_{0}^{t}\|\Delta u\|_{L^{2}}^{2} d \tau \\
& +C\left(\int_{0}^{t}\left\|u_{3}\right\|_{L^{\alpha}}^{\frac{2 \alpha}{\alpha-3}}\|\nabla u\|_{L^{2}}^{2} d \tau\right)^{\frac{4(\alpha-3)}{3 \alpha-10}} \\
& +C\left(\int_{0}^{t}\|\nabla b\|_{\dot{M}_{p, q}}^{\frac{2 p}{2 p-3}}\|\nabla b\|_{L^{2}}^{\frac{2 p}{2 p-3}}\|\nabla u\|_{L^{2}}^{\frac{2(p-3)}{2 p-3}} d \tau\right)^{\frac{2(2 p-3)}{3(p-2)}} \\
& \leq C\left(\left\|\nabla_{h} u_{0}\right\|_{L^{2}}^{\frac{8}{3}}+\left\|\nabla_{h} b_{0}\right\|_{L^{2}}^{\frac{8}{3}}+1\right)+\frac{3}{4} \int_{0}^{t}\|\Delta u\|_{L^{2}}^{2} d \tau \\
& +C\left(\int_{0}^{t}\left\|u_{3}\right\|_{L^{\alpha}}^{\frac{2 \alpha}{\alpha-3}}\|\nabla u\|_{L^{2}}^{2} d \tau\right)^{\frac{4(\alpha-3)}{3 \alpha-10}} \\
& +C\left(\int_{0}^{t}\|\nabla b\|_{\dot{M}_{p, q}}^{\frac{2 p}{2 p-3}}\left(\|\nabla b\|_{L^{2}}^{2}+\|\nabla u\|_{L^{2}}^{2}\right) d \tau\right)^{\frac{2(2 p-3)}{3(p-2)}} \\
& \leq C\left(\left\|\nabla_{h} u_{0}\right\|_{L^{2}}^{\frac{8}{3}}+\left\|\nabla_{h} b_{0}\right\|_{L^{2}}^{\frac{8}{3}}+1\right)+\frac{3}{4} \int_{0}^{t}\|\Delta u\|_{L^{2}}^{2} d \tau \\
& +C\left(\int_{0}^{t}\left\|u_{3}\right\|_{L^{\alpha}}^{\frac{2 \alpha}{\alpha-3}}\|\nabla u\|_{L^{2}}^{\frac{3 \alpha-10}{2(\alpha-3)}}\|\nabla u\|_{L^{2}}^{\frac{\alpha-2}{2(\alpha-3)}} d \tau\right)^{\frac{4(\alpha-3)}{3 \alpha-10}} \\
& +C\left(\int_{0}^{t}\|\nabla b\|_{\dot{M}_{p, q}}^{\frac{2 p}{2 p-3}}\left(\|\nabla b\|_{L^{2}}^{\frac{3(p-2)}{2 p-3}}+\|\nabla u\|_{L^{2}}^{\frac{3(p-2)}{2 p-3}}\right)\left(\|\nabla b\|_{L^{2}}^{\frac{p}{2 p-3}}+\|\nabla u\|_{L^{2}}^{\frac{p}{2 p-3}}\right) d \tau\right)^{\frac{2(2 p-3)}{3(p-2)}} \\
& \leq C\left(\left\|\nabla_{h} u_{0}\right\|_{L^{2}}^{\frac{8}{3}}+\left\|\nabla_{h} b_{0}\right\|_{L^{2}}^{\frac{8}{3}}+1\right)+\frac{3}{4} \int_{0}^{t}\|\Delta u\|_{L^{2}}^{2} d \tau \\
& +C\left(\int_{0}^{t}\left\|u_{3}\right\|_{L^{\alpha}}^{\frac{8 \alpha}{3 \alpha-10}}\|\nabla u\|_{L^{2}}^{2} d \tau\right)\left(\int_{0}^{t}\|\nabla u\|_{L^{2}}^{2} d \tau\right)^{\frac{\alpha-2}{3 \alpha-10}} \\
& +C\left(\int_{0}^{t}\|\nabla b\|_{\dot{M}_{p, q}}^{\frac{4 p}{3(p-2)}}\left(\|\nabla b\|_{L^{2}}^{2}+\|\nabla u\|_{L^{2}}^{2}\right) d \tau\right)\left(\int_{0}^{t}\|\nabla b\|_{L^{2}}^{2}+\|\nabla u\|_{L^{2}}^{2} d \tau\right)^{\frac{p}{3(p-2)}} \\
& \leq C\left(\left\|\nabla_{h} u_{0}\right\|_{L^{2}}^{\frac{8}{3}}+\left\|\nabla_{h} b_{0}\right\|_{L^{2}}^{\frac{8}{3}}+1\right)+\frac{3}{4} \int_{0}^{t}\|\Delta u\|_{L^{2}}^{2} d \tau \\
& +C \int_{0}^{t}\left(\left\|u_{3}\right\|_{L^{\alpha}}^{\frac{8 \alpha}{3 \alpha-10}}+\|\nabla b\|_{\dot{M}_{p, q}}^{\frac{4 p}{3(p-2)}}\right)\left(\|\nabla u\|_{L^{2}}^{2}+\|\nabla \omega\|_{L^{2}}^{2}+\|\nabla b\|_{L^{2}}^{2}\right) d \tau .
\end{aligned}
$$

Inserting the above inequality into (4.20), we have

$$
\begin{aligned}
& \left(\|\nabla u\|_{L^{2}}^{2}+\|\nabla \omega\|_{L^{2}}^{2}+\|\nabla b\|_{L^{2}}^{2}\right)+\int_{0}^{t}\left(\|\Delta u\|_{L^{2}}^{2}+\|\Delta \omega\|_{L^{2}}^{2}\right) d \tau \\
& \quad \leq C\left(\left\|\nabla u_{0}\right\|_{L^{2}}^{2}+\left\|\nabla \omega_{0}\right\|_{L^{2}}^{2}+\left\|\nabla b_{0}\right\|_{L^{2}}^{2}+1\right)
\end{aligned}
$$




$$
\begin{aligned}
& +C \int_{0}^{t}\left(\|\nabla b\|_{\dot{M}_{p, q}}^{\frac{2 p}{2 p-3}}+\left\|\nabla|\omega|^{\frac{3}{2}}\right\|_{L^{2}}^{2}+\left\|u_{3}\right\|_{L^{\alpha}}^{\frac{8 \alpha}{3 \alpha-10}}+\|\nabla b\|_{\dot{M}_{p, q}}^{\frac{4 p}{3(p-2)}}\right) \\
& \times\left(\|\nabla u\|_{L^{2}}^{2}+\|\nabla \omega\|_{L^{2}}^{2}+\|\nabla b\|_{L^{2}}^{2}\right) d \tau,
\end{aligned}
$$

Gronwall's inequality and Lemma 3.1 help to obtain

$$
\begin{aligned}
& \left(\|\nabla u\|_{L^{2}}^{2}+\|\nabla \omega\|_{L^{2}}^{2}+\|\nabla b\|_{L^{2}}^{2}\right)+\int_{0}^{t}\left(\|\Delta u\|_{L^{2}}^{2}+\|\Delta \omega\|_{L^{2}}^{2}\right) d \tau \\
& \leq C\left(\left\|\nabla u_{0}\right\|_{L^{2}}^{2}+\left\|\nabla \omega_{0}\right\|_{L^{2}}^{2}+\left\|\nabla b_{0}\right\|_{L^{2}}^{2}+1\right) \\
& \quad \times \exp \left\{\int_{0}^{T}\left(\|\nabla b\|_{\dot{M}_{p, q}}^{\frac{2 p}{2 p-3}}+\left\|\nabla|\omega|^{\frac{3}{2}}\right\|_{L^{2}}^{2}+\left\|u_{3}\right\|_{L^{\alpha}}^{\frac{8 \alpha}{3 \alpha-10}}+\|\nabla b\|_{\dot{M}_{p, q}}^{\frac{4 p}{3(p-2)}}\right) d \tau\right\} \\
& \leq C\left(\left\|\nabla u_{0}\right\|_{L^{2}}^{2}+\left\|\nabla \omega_{0}\right\|_{L^{2}}^{2}+\left\|\nabla b_{0}\right\|_{L^{2}}^{2}+1\right) \\
& \quad \times \exp \left\{C \int_{0}^{T}\left(1+\left\|u_{3}\right\|_{L^{\alpha}}^{\frac{8 \alpha}{3 \alpha-10}}+\|\nabla b\|_{\dot{M}_{p, q}}^{\frac{4 p}{3(p-2)}} d \tau\right\}\right.
\end{aligned}
$$

which completes the proof of Theorem 1.1.

\section{Acknowledgements}

This work was supported by the National Natural Science Foundation of China (No. 11961032 and No. 11971209), the Natural Science Foundation of Jiangxi Province, China (No. 20191BAB201003).

\section{Availability of data and materials}

Not applicable.

\section{Competing interests}

The authors declare that they have no competing interests.

\section{Authors' contributions}

The authors declare that the study was realized in collaboration with the same responsibility. All authors read and approved the final manuscript.

\section{Publisher's Note}

Springer Nature remains neutral with regard to jurisdictional claims in published maps and institutional affiliations.

Received: 16 March 2021 Accepted: 21 June 2021 Published online: 15 July 2021

\section{References}

1. Cao, C., Wu, J.: Two regularity criteria for the 3D MHD equations. J. Differ. Equ. 248, 2263-2274 (2010)

2. Gala, S.: Regularity criteria for the $3 D$ magneto-microploar fluid equations in the Morrey-Campanato space. Nonlinear Differ. Equ. Appl. 17, 181-194 (2010)

3. Gala, S., Ragusa, M.A.: A logarithmic regularity criterion for the two-dimensional MHD equations. J. Math. Anal. Appl. $444,1752-1758$ (2016)

4. Gala, S., Ragusa, M.A.: On the regularity criterion of weak solutions for the 3D MHD equations. Z. Angew. Math. Phys. $68,140(2017)$

5. Gala, S., Ragusa, M.A.: A new regularity criterion for the 3D incompressible MHD equations via partial derivatives. J. Math. Anal. Appl. 481, 123497 (2020)

6. Gala, S., Ragusa, M.A., Ye, Z.: An improved blow-up criterion for smooth solutions of the two-dimensional MHD equations. Math. Methods Appl. Sci. 40, 279-285 (2017)

7. Galdi, G.P., Rionero, S.: A note on the existence and uniqueness of solutions of the microploar fluid equations. Int. J. Eng. Sci. 15, 105-108 (1997)

8. Jia, X., Zhou, Y.: Regularity criteria for the 3D MHD equations involving partial components. Nonlinear Anal., Real World Appl. 13, 410-418 (2012)

9. Jia, X., Zhou, Y.: Regularity criteria for the 3D MHD equations via partial derivatives. Kinet. Relat. Models 5, 505-516 (2012)

10. Lemarié-Rieusset, P.G.: The Navier-Stokes equations in the critical Morrey-Campanato space. Rev. Mat. Iberoam. 23, 897-930 (2007)

11. Lukaszewicz, G.: Micropolar Fluids: Theory and Applications. Birkhäuser, Berlin (1998)

12. Ortega-Torres, E.E., Rojas-Medar, M.A.: Magneto-microploar fluid motion: global existence of strong solutions. Abstr. Appl. Anal. 4, 109-125 (1999) 
13. Rojas-Medar, M.A.: Magneto-microploar fluid motion: existence and uniqueness of strong solutions. Math. Nachr. 188, 301-319 (1997)

14. Rojas-Medar, M.A., Boldrini, J.L.: Magneto-microploar fluid motion: existence of weak solutions. Internet Rev. Mat. Complut. 11, 443-460 (1998)

15. Yamazaki, K.: Regularity criteria of MHD system involving one velocity and one current density component. J. Math. Fluid Mech. 16, 551-570 (2014)

16. Zhang, Z: Regularity criteria for the $3 \mathrm{D}$ MHD equations involving one current density and the gradient of one velocity component. Nonlinear Anal. 115, 41-49 (2015)

17. Zhang, Z., Yao, Z., Wang, X.: A regularity criterion for the 3D magneto-micropolar fluid equations in Triebel-Lizorkin spaces. Nonlinear Anal. 74, 2220-2225 (2011)

18. Zhou, Y., Fan, J.: A regularity criterion for the $2 \mathrm{D}$ MHD system with zero magnetic diffusivity. J. Math. Anal. Appl. 378 169-172 (2011)

Submit your manuscript to a SpringerOpen ${ }^{\circ}$ journal and benefit from:

- Convenient online submission

Rigorous peer review

- Open access: articles freely available online

- High visibility within the field

- Retaining the copyright to your article

Submit your next manuscript at $\gg$ springeropen.com 